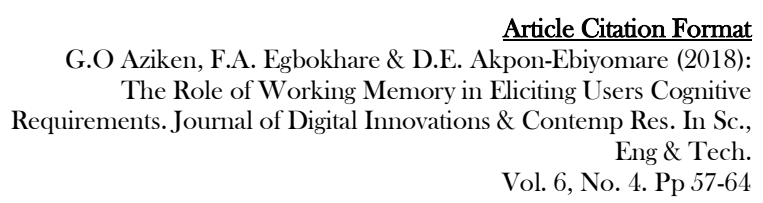

Article Citation Format

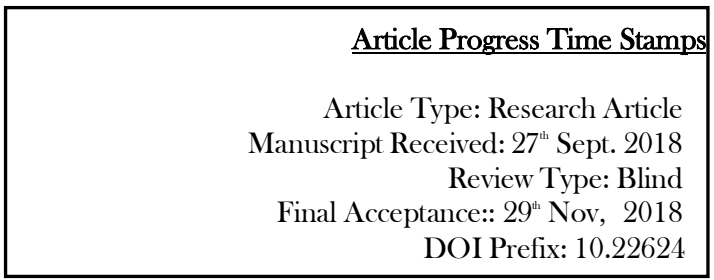

\title{
The Role of Working Memory in Eliciting Users Cognitive Requirements
}

\author{
G.O Aziken, F.A. Egbokhare \& D.E. Akpon-Ebiyomare \\ Department of Computer Science \\ University of Benin \\ Benin City, Nigeria \\ Email: goaziken@uniben.edu
}

\begin{abstract}
Requirements elicitation is the initial phase of requirements engineering concerned with gathering users' needs, which makes it a major determinant of the outcome of the entire software development process. Software users have psychological concerns which influence requirements elicitation and a knowledge of these cognitive processes will enable developers empathize with users during requirements elicitation and enable them capture their cognitive needs which are formulated as mental models. This research conducted experiments to determine the role of working memory in the elicitation of users' cognitive requirements. Findings showed that the best place to capture users' implicit needs is from the working memory when a users' mind is formulating mental models along the line of the solution to the problem. This will improve the requirements engineering process, better project outcomes and more satisfied users.
\end{abstract}

Keywords: Requirements elicitation, working memory, software development, users cognitive needs

\section{INTRODUCTION}

Satisfying users exact needs is the goal of every software development project but rework due to the inability of developed software systems to meet users' expectation is currently a burning issue in software development research (Adnan and Naqvi, 2015; MicroFocus, 2010; Ellis, 2009). Software Requirements engineering is associated with several challenges resulting from the inability of the users to express their exact needs (Swarnalatha et al., 2014; Hagal and Kandemili, 2017). Robertson (2001) observed that what users desire in a software system is sometimes tacit and difficult to express but is formulated in the form of mental models. Most software project managers perceive the requirements elicitation activity as a social process and thus prescribe elicitation techniques that encourage social interactions between the software developers and the users. This has resulted in the development of systems with incomplete requirements because the users cognitive needs are excluded (Aziken and Egbokhare, 2017). While Scientists (Lynam et al., 2012) argued that the mind constructs mental models as a result of knowledge, perception and imagination, Johnson-Laird (1983) viewed mental models as temporary working memory sketches set up for immediate purposes. 
The working memory is a unit that maintains, controls and manipulates a limited amount of information before recall (Baddeley, 2003). This research conducted experiments to determine the role of working memory in the elicitation of users' cognitive requirements.

\subsection{Background of Study}

The working memory contains three main sections: Short term memory, executive centre and attention system. It acts as an interface between perception, long term memory and action (Figure 1).

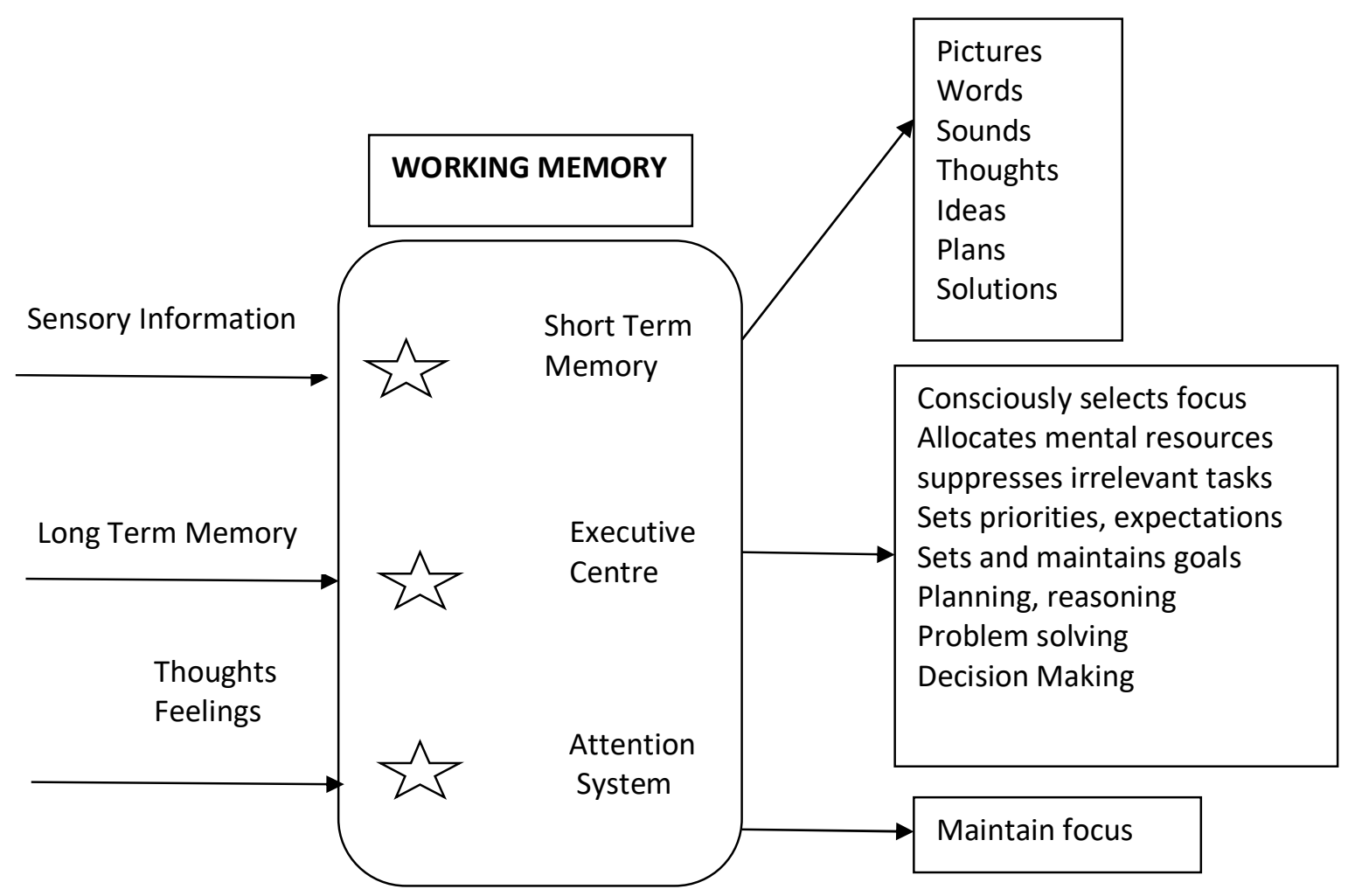

Figure 1: The Working Memory

The Working memory has been a subject of various studies related to cognition $(\mathrm{Ku}, 2018$; Bays, 2018; Borst et al., 2012; Parra et al., 2014), which is central to many aspects of intelligent human life (Conway et al., 2002; 2003). It creates a mental workspace to hold information temporarily while at the same time carrying out other cognitive processes related to perception, decision making, problem solving and reasoning (Lepsien et al., 2005; Johnson et al., 2007; Soto et al., 2007). The mental work space available determines the ability of the working memory to respond to relevant information and discard unnecessary ones hence cognition depends heavily on it (Bleddowski et al., 2009). Eliasmith, (2001) opined that working memory gives meaning to the world that is perceived, since it processes information based on perception and makes it ready for goal directed actions (Baddeley, 2012). Sources of information into the working memory are either external in which case it is received from human sensory systems (Chen et al., 2006) or internal when the information is from the long term memory (Song and Jiang, 2005). 
Permanent information resides in the long term memory and is dormant until when it is recalled or fetched back into consciousness (Abbot, 2002). The working memory is limited in capacity (Ma et al., 2014; Luck and Vogel, 2013). According to Miller (1956), an individual normally has the ability to process or retain $7 \pm 2$ chunks of information in their correct serial order. Cowan (2000) later suggested that the capacity limit of working memory was between 3 and 5 chunks, while Caruthers (2013) opined that the working memory holds three to four chunks of information at any time. Chunking is a psychological process through which individual pieces of information are bound together into a meaningful whole (Neath and Surprenant, 2003). This limitation in the capacity of the working memory is the reason people feel overwhelmed by information when there is information overload (Cowan, 2005).

The working memory has a short span (Kaczmarzyk et al., (2013) thus information in it can only be captured if necessary attention is paid to it as a "to-be-remembered" information before it undergoes decay (Awh and Jonides, 2001). Findings from Bears (2002) show that the information in the working memory decays within 15 - 30 seconds, thus capturing users cognitive needs that are formulated in the form of mental models requires that it should be done while still in the working memory. Another characteristic of the working memory is that it is associated with core cognitive processes which includes perception, problem solving, behaviours and action control. Postle (2006) and Cowan et al. (2005) opined that the working memory functions mainly by controlled attention because attention enhances task performance in the working memory (Clapp et al., 2011; Clapp and Gazzaley, 2012), hence the brain manages information in the working memory by selectively replacing its content with newly received information and displacing the irrelevant ones which are no longer active (Leung et al., 2007; Roth and Courtney, 2007). The working memory and selective attention are described as related but independent core cognitive functions (Jia et al., 2017; Myers et al., 2017; Souza and Oberaurer, 2016). Selective attention is critical to information processing, because it ensures that there is no information overload by allocating available resources to the most important and critical aspect of the information being processed.

\section{METHODOLOGY}

An experimental method was used in this study. A quasi experiment involving two groups was conducted on students preparing to write a computer based test (CBT) at the University of Benin, Nigeria. The aim was to determine the requirements for a system to conduct an online multiple choice objective examination. The first step in the experiment was to categorize the users based on criteria proposed in (Aziken and Egbokhare 2018). Two students from each category were selected for the experiment. A prototype system was developed for the experiment. The first screen contained three multi choice questions with the caption "PREVIOUS' and "NEXT" to direct the student to the previous on next screen. The second screen had only one question with the font size larger than the first and the arrows " previous and next ". The third Screen had one question but it automatically drops down after a minute into the next question. Also there was a "SUBMIT" button and Press "PgDn" or "PgUp" to go to the next or previous screen. The first test was conducted on four students representing each category. .Each student was assigned a computer system and asked to answer the questions. At the end of the session, the students were asked to give useful feedback that could be used to update the system. One of the authors also observed the students' reaction to each screen type.

The second group of students were tested individually using "think Aloud" protocol. Each student was asked to answer the questions and to "Speak Out" whatever came to their mind as they used the system. A recorder was placed by each student and also like most protocol verbalization experiments, one of the authors observed to ensure that they did not keep quiet for too long. 


\section{FINDINGS/DISCUSSION}

Ten minutes after the experiment, the first set of students were interviewed to determine their feedbacks to enhance the system. The interview was in the form of a focus group discussion which lasted for ten (10) minutes. The feedback obtained were:

1. Interface was not exciting. We need screens that are colourful and bright

2. Whether single or multiple questions per screen, clarity is the major concern

3. We the users are never carried along when systems that concern us are being built. For example look at the Portal that was introduced without carrying students along.

4. That message box popped up too often. It was even blocking the questions. That's how viruses are introduced into programs

5. Initially, I did not understand the meaning of " $\Rightarrow \rightleftarrows$ ". I prefer instructions in simple English.

6. I should be given the option of choosing how I want my questions arranged

7. My screen was just somehow.

8. I did not like the way the last question just dropped down. I was not through with the previous screen. That's why we should be carried along and given a brief training. Management does not always think of us the students

Some verbalizations obtained from the think aloud Protocol include:

1. Why are the fonts so small?

2. Oh oo. This thing is wasting my time. The mouse is just jumping across the screen

3. Has this system hanged? Abi it's the keyboard?

4. Thank God

5. En hen. This is better. At least the font is larger and clearer

6. Thank God this is not the main exam. This message is blocking the questions

7. Aaaa. I did not click anything. How did I get to this screen?

8. Excuse me. How do I go to the next screen? Which keyooooo

9. Oooo. Which one is this key again? Abeg the instructions are too many

10. The time is too short

The role of selective attention in displacing the content of working memory when new information emerge was observed during the interview session. The requirements gathered were not comprehensive enough as participants talked at random and deviated often from the subject matter thereby leaving out some important information. Users had difficulties expressing their observations because other information from the permanent memory has been processed and their immediate attention was no longer focused on the prototype.

The responses obtained from the verbalization experiment were analysed in relation to the aim of the experiment. It was discovered that when users speak out as they encounter problems, the exact mental models are captured and this can be combined with other requirements to produce more complete systems. For example, "Oooo. Which one is this key again? Abeg the instructions are too many" indicates that the user is having difficulties locating a particular key on the keyboard. Also, the exclamation "I did not press any key indicates that the user prefers to be in charge of how the next question appears. User satisfaction is key to success in software development projects. Software developers can address lapses in user satisfaction by involving and communicating with users throughout the software project. Poor knowledge of users which results from poor communication leads to situations where software applications are developed for users to adapt to instead of the system being adapted to the users, which creates conflict between developers and users due to user dissatisfaction. 
Ideas, observations and comments from users especially during requirements elicitation should not be ignored because they have the domain knowledge needed to handle the problems the software application is designed to solve. Software products should be easy to use and not cause increased cognitive load for users. When users put in so much effort to work with a software, they abandon it within a short time leading to failure of the project.

\section{CONCLUSION}

Incomplete requirements have been identified as a major cause of failures in software development projects. This is associated with challenges encountered by users while expressing their cognitive needs which are tacit in nature and resides in their minds. Software users have psychological concerns which influence requirements elicitation and a knowledge of these cognitive processes will enable developers empathize with users during requirements elicitation by stimulating them to think and formulate useful mental models that uncover their exact needs. Capturing the cognitive needs as they occur from the working memory produces more accurate models that represent the users' cognitive needs. The more software developers understand this concept and the nature of human consciousness experience, the more they will view users problems beyond technical perspectives. 


\section{REFERENCES}

1. Abbot, B. (2002). Human memory. Fort Wayne: Indiana University-Purdue University at Fort Wayne, Psychology Department. Retrieved July 042018 from http://www.users.ipfw.edu/abbot

2. Adnan, F. and Naqvi, I. H. (2015). Effect of rework on project success. Science International (Lahore). ISSN 1013-5316; CODEN: SINTE 8: 27(1), 575-580

3. Awh and Jonides (2001).Overlapping mechanisms of attention and spatial working memory. Trends Cogn Sci.; 5:119-126.

4. Aziken, G. and Egbokhare, F. (2017a). The role of Cognitive Psychological Processes in Software Requirements Elicitation. Journal of the Nigerian Association of Mathematical Physics. Vol. 41. 352-358

5. Baddeley, A. (2003). Working Memory: looking back and looking forward. Nature Review Neuroscience, 4, 829 - 839

6. Baddeley, A. (2012). Working memory: theories, models, and controversies. Annual Review of Psychology. 2012;63:1-29.

7. Bays, P. M. (2018). Reassessing the evidence for capacity limits in neural signals related to working memory. Cerebral Cortex 28:1432-1438

8. Bays, P. M., Catalao, R. F. G. and Husain, M. (2009). The precision of visual working memory is set by allocation of a shared resource. Journal of Vision, 9(10), Article 7. Retrieved July 04 2018 from http://www.journalofvision.org/content/9/10/7

9. Bears, B. J. (2002). The conscious access hypothesis: Origins and recent evidence. Trends in Cognitive Science. 6 (1), 47 - 52

10. Bleddowski, C., Rahm, B. and Rowe, J. B. (2009). The Journal of Neuroscience. 29(43):1373513741

11. Borst, G., Niven, E. H. and Logie, R. H. (2012). Visual mental Image generation does not overlap with visual short-term memory: A dual task interference study. Memory and Cognition, 40, 360-372

12. Caruthers, P. (2013). Evolution of working memory. PNAS 110 (2). 10371 - 10378 Retrieved 24 August 2018 from, www.nas.org/cgi/doi/10.1073/pnas.1301195110

13. Chen, A. J., Abrams, G. M. and D'Esposito, M. (2006). Functional reintegration of prefrontal neural networks for enhancing recovery after brain injury. J Head Trauma Rehabil; 21: 107-18.

14. Clapp, W. C., Rubens, M. T., Sabharwal, J. and Gazzaley, A. (2011). Deficit in switching between functional brain networks underlies the impact of multitasking on working memory in older adults. Proceedings of the National Academy of Sciences of the United States of America; 108:7212-7217

15. Clapp, W. C. and Gazzaley, A. (2012). Distinct mechanisms for the impact of distraction and interruption on working memory in aging. Neurobiology of Aging.;33:134-148

16. Cowan, N. (2000). The magical number 4 in short-term memory: A reconsideration of mental storage capacity. Behavioural and Brain Sciences. 24, 87-185

17. Conway, A. R. A., Cowan, N., Bunting, M. F., Therriault, D. J., and Minkoff, S. R. B. (2002). A latent variable analysis of working memory capacity, short-term memory capacity, processing speed, and general fluid intelligence. Intelligence, 30, 163-183.

18. Conway, A. R. A., Kane, M. J. and Engle, R. W. (2003). Working memory capacity and its relation to general intelligence. Trends Cogn. Sci. (Regul. Ed.) 7, 547-552

19. Cowan N. (2005). Working memory capacity. Hove, East Sussex, UK: Psychology Press 
20. Cowan, N., Elliot, E. M., Scott, S. J., Morey, C. C., Mattox, S., Hismjatullina, A. and Conway, A. R. (2005). On the capacity of attention: its estimation and its role in

21. working memory and cognitive aptitudes. Cognitive Psychology, 51 (1): 42 -100

22. Eliasmith, C. (2001). Memory. Dictionary of philosophy of mind. Pullman, WA: Washington State University.Retrieved July 04 2018 from http://www.artsci.wustl.edu/ ${ }^{\sim}$ philos/minddict/memory.html

23. Ellis, K. (2009). The path to success. IAG Consulting. Business Analysis Benchmark

24. Hagal, M. and Kandemili, F. (2017). Reducing Missed Requirements Issues: Complete, Unambiguous and Necessary Requirements Elicitation. International Journal of Advanced Research in Computer Science and Software Engineering. 7(1), 10-14

25. Jia, J., Liu, L., Fang, F. and Luo, H. (2017). Sequential sampling of visual objects during sustained attention. PLOS Biology.;15:e2001903

26. Johnson, M. R., Mitchell K. J., Raye, C. L., D'Esposito, M. and Johnson, M. K. (2007). A brief thought can modulate activity in extrastriate visual areas: Top-down effects of refreshing just-seen visual stimuli. Neuroimage.; 37:290-299.

27. Johnson-Laird, P. (1983). Mental models: Towards a cognitive science of language, inference, and consciousness. Cambridge, MA: Harvard University Press.

28. Kaczmarzyk, M., Francikowski, J., Łozowski, B., Rozpędek, M., Sawczyn, T. and Sułowicz, S. (2013). The bit value of Working Memory. Psychology and Neuroscience. 6, 3, 345-349

29. Ku, Y. (2018). Selective attention on representations in working memory: cognitive and neural mechanisms. DOI 10.7717/peerj.4585. 1-16

30. Lepsien, J, Griffin, I. C., Devlin, J. T. and Nobre, A. C. (2005). Directing spatial attention in mental representations: Interactions between attentional orienting and working-memory load. Neuroimage. ; 26:733-743

31. Leung, H.C., Oh, H., Ferri J. and Yi, Y. (2007). Load response functions in the human spatial working memory circuit during location memory updating. Neuroimage.; 35:368-377.

32. Luck, S. J. and Vogel, E. K. (2013). Visual working memory capacity: from psychophysics and Neurobiology to individual differences. Trends in Cognitive Sciences 17:391-400

33. Lynam, T., Mathevet, R., Etienne, M., StoneJovicich, S., Leitch, A., Jones, N., Ross, H., Du Toit, D., Pollard, S., Biggs, H. and Perez, P. (2012). Waypoints on a journey of Discovery: Mental Models in human environment interactions. Ecology and Society: a journal of Integrative Science for resilience and Sustainability, 17, 23 - 33

34. Ma, W. J., Husain, M. and Bays, P. M. (2014). Changing concepts of Working Memory. Nature Review Neuroscience, 17(3), 347 - 356

35. MicroFocus. (2010). Successful Projects start with high quality requirements, 1-10

36. Miller, G. A. (1956). The magical number seven, plus or minus two: some limits on our capacity for processing information. Psychol. Rev.;63:81-97

37. Myers, N. E., Stokes, M. G. and Nobre, A. C. (2017). Prioritizing information during working memory: beyond sustained internal attention. Trends in Cognitive Sciences.;21:449-461.

38. Neath I. and Surprenant A. (2003). Human memory. 2nd ed. Belmont, CA: Wadsworth.

39. Parra, M. A., Della Sala, S., Logie, R. H. and Morcom, A. (2014). Neural correlates of shape-color binding in visual working memory. Neuropsychologia, 52C, 27-36

40. Postle, P. R. (2006). Working Memory as an emergent property of the mind and brain. Neuroscience 139(1), 23 - 38

41. Robertson, S. (2001). Requirements Trawling: Techniques for discovering requirements. International Journal of Human Computer Studies. 55(4), 405-421 
42. Roth, J. K. and Courtney, S. M. (2007). Neural system for updating object working memory from different sources: Sensory stimuli or long-term memory. Neuroimage.; 38:617-630.

43. Song, J. H. and Jiang Y. (2005). Connecting the past with the present: how do humans match an incoming visual display with visual memory? Journal of Vision, 5, 322-330.

44. Soto, D., Humphreys, G. W. and Rotshtein, P. (2007). Dissociating the neural mechanisms of memory-based guidance of visual selection. Proc Natl Acad Sci U S A.; 104:17186-17191.

45. Souza, A. S. and Oberauer, K. (2016). In search of the focus of attention in working memory: 13 years of the retro-cue effect. Attention, Perception \& Psychophysics.;78:1839-1860

46. Swarnalatha, K.S., Srinivasan, G.N., Meghana, D., Raunak, K. and Kopal S. (2014). A Survey on Software Requirement Engineering for Real Time Projects based on Customer Requirement. International Journal of Advanced Research in Computer and Communication Engineering. 3(1), $5045-5050$ 